\title{
Effect of pressure on the phase diagrams and dynamic properties of easy-plane antiferromagnets
}

\author{
Yu. N. Mitsay, Yu. A. Fridman, G. A. Bairamaliyeva, and C. N. Alexeev
}

Simferopol State University, 333036, Simferopol, Yaltinskaya st., 4

Submitted March 19, 1997, revised May 22, 1997

\begin{abstract}
Phase diagrams and dynamic properties of strongly anisotropic, easy-plane antiferromagnets in the presence of external pressure are studied. It is demonstrated that the phase with tensorial order parameter is possible in the system. Spectra of bound magnetoelastic waves are studied in the vicinity of the phase transition points.
\end{abstract}

PACS: $75.50 . \mathrm{Ee}$

\section{Introduction}

Special attention has recently been focused on the study of peculiarities of the behavior of easyplane magnets with one-ion anisotropy [1-3]. Systems with the energy of uniaxial anisotropy comparable or greater than the energy of the exchange interaction have attracted considerable interest. First of all, it is connected with the fact that such magnets are low-temperature magnets. They are $[4,5]$

$\mathrm{NiSiF}_{6} \cdot 6 \mathrm{H}_{2} \mathrm{O}, \quad \mathrm{NiZrF}_{6} \cdot 6 \mathrm{H}_{2} \mathrm{O}, \quad \mathrm{FeSiF}_{6} \cdot 6 \mathrm{H}_{2} \mathrm{O}$, $\mathrm{CsFeCl}_{3}$, and some others. The behavior of these magnets in a transverse magnetic field has been studied extensively $[1,3]$ and interesting peculiarities of their properties, such as the emergence of the quadruple phase (QU) and discontinuity of phase diagrams when the value of the magnetic ion spin is greater than unity, have been revealed. The dynamical properties of such systems have also been studied extensively in the vicinity of the orientational phase transition (OPT).

However, the behavior of such magnets in a longitudional field (parallel to the basic plane) has been studied insufficiently since investigations of this case were limited to small anisotropy $(\beta<<J$, where $\beta$ is the constant of one-ion anisotropy, and $J$ is the exchange constant) [6]. The influence of mechanical boundary conditions on the properties of strongly anisotropic easy-plane magnets and spectra of elementary excitations with allowance for magnetoelastic interaction have virtually been ignored in those studies.

In this study we have focused our attention on these factors.

\section{Phase diagram of a strongly anisotropic easy-plane antiferromagnet}

The system under study is a strongly anisotropic $(\beta>>J)$ antiferromagnet with the Dzyaloshinski's exchange. The resulting magnetic momentum lies in the easy plane $(X Y)$, as does the applied external magnetic field. Let us determine the influence of the external pressure on the static and dynamic properties of the magnet. We assume that the external pressure is applied parallel to the easy plane. As we shall see, this is the most interesting case.

The Hamiltonian of the system has the form

$$
\begin{aligned}
\mathcal{H}_{0} & =-H \sum_{n_{i}} S_{n_{i}}^{x}+\frac{\beta}{2} \sum_{n}\left(S_{n_{i}}^{z}\right)^{2}+\sum_{n_{1}, n_{2}}\left\{J\left(n_{1}-n_{2}\right) \mathbf{S}_{n_{1}} \mathbf{S}_{n_{2}}-D\left(n_{1}-n_{2}\right)\left[\mathbf{S}_{n_{1}} \times \mathbf{S}_{n_{2}}\right]_{z}\right\}+v \sum_{n_{i}} S_{n_{i}}^{j} S_{n_{i}}^{k} u_{j k}\left(n_{i}\right)+ \\
& +\int d r\left\{\frac{\lambda+\eta}{2}\left(u_{x x}^{2}+u_{y y}^{2}+u_{z z}^{2}\right)+\eta\left(u_{x y}^{2}+u_{x z}^{2}+u_{y z}^{2}\right)+\lambda\left(u_{x x} u_{y y}+u_{y y} u_{z z}+u_{x x} u_{z z}\right)-P u_{x x}\right\},
\end{aligned}
$$




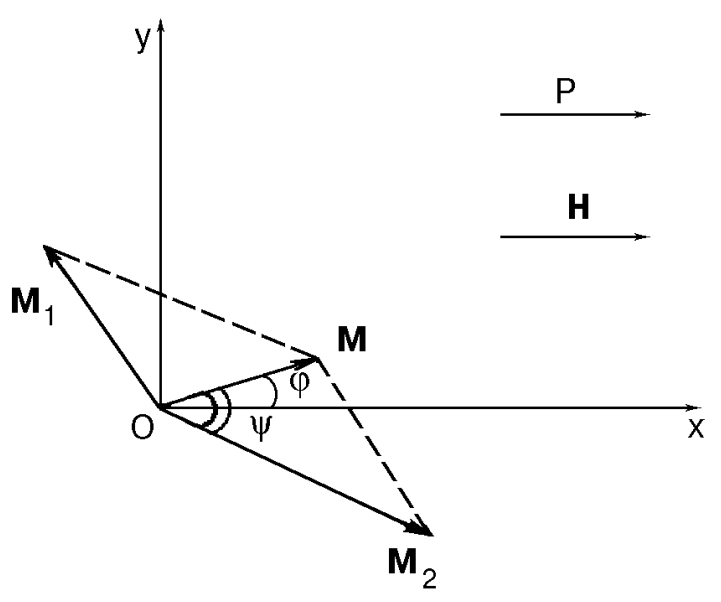

Fig. 1. Orientation of the sublattice magnetic momenta.

where $S_{n_{i}}^{\alpha}$ is the spin operator at site $n$ of the $i$ th sublattice, $D\left(n_{1}-n_{2}\right)<0$ the Dzyaloshinski's exchange, $v$ is the constant of magnetoelastic (ME) exchange, $\lambda$ and $\eta$ are the elasticity moduli, $u_{j k}\left(n_{i}\right)$ is the deformation tensor, $J\left(n_{1}-n_{2}\right)>0$ is the exchange integral, $P$ is the external pressure, and $H$ is the external magnetic field.

In (1) the first three terms describe the magnetic subsystem, the fifth term describes the elastic subsystem, and the fourth term describes the $\mathrm{ME}$ exchange. Further investigations are carried out in a low-temperature limit $\left(T \ll T_{N}, T_{N}\right.$ is the Néel temperature) since in this case it is possible to make all calculations analytically. Without lack of generality we can assume the magnetic ion spin to be $S=1$. The orientation of the sublattice magnetic momenta for the system described by (1) can be represented as shown in Fig. 1.

Rotate the coordinate system around the axis (perpendicular to the plane of Fig. 1) so that the new quantization axis $\xi_{i}$ would be parallel to the vector of magnetization of the $i$ th sublattice. In this local coordinate system define the new spin operators $S_{n_{i}}^{\xi}, S_{n_{i}}^{\eta}$, and $S_{n_{i}}^{\zeta}$ as follows:

$$
\begin{gathered}
S_{n_{i}}^{x}=S_{n_{i}}^{\xi} \cos \theta_{i}+(-1)^{i+1} S_{n_{i}}^{\eta} \sin \theta_{i}, \\
S_{n_{i}}^{y}=(-1)^{i+1} S_{n_{i}}^{\xi} \sin \theta_{i}+S_{n_{i}}^{\eta} \cos \theta_{i}, \\
S_{n_{i}}^{z}=S_{n_{i}}^{\zeta}, \quad \psi=\frac{\theta_{1}+\theta_{2}}{2}, \quad \varphi=\frac{\theta_{1}-\theta_{2}}{2} .
\end{gathered}
$$

We carry out further calculations with use of the Hubbard's operators $[3,6]$, which make it possible to exactly take into account the one-ion and $\mathrm{ME}$ exchange. These operators are built on eigenfunctions of the one-site Hamiltonian $\mathcal{H}_{0}\left(n_{i}\right)$. After separation of the mean field in the exchange part of (1) and transition to local operators (2) the one-site Hamiltonian assumes the form

$$
\begin{gathered}
\mathcal{H}_{0}=-\sum_{n_{i}} H_{i} S_{n_{i}}^{\xi}+\sum_{n_{i}}(-1)^{i+1} \bar{H}_{i}\left(S_{n_{i}}^{+}+S_{n_{i}}^{-}\right)-\frac{\beta}{8} \sum_{n_{i}}\left\{\left(S_{n_{i}}^{+}\right)^{2}+\left(S_{n_{i}}^{-}\right)^{2}-S_{n_{i}}^{+} S_{n_{i}}^{-}-S_{n_{i}}^{-} S_{n_{i}}^{+}\right\}+\frac{v}{2} \sum_{n_{i}}\left\{\left(u_{x x}^{i}+u_{y y}^{i}-u_{z z}^{i}-\right.\right. \\
\left.\left.-A_{i}+B_{i}\right)\left(S_{n_{i}}^{+}\right)^{2}+2 A_{i}\left(S_{n_{i}}^{\xi}\right)^{2}+\frac{1}{2}\left(u_{x x}^{i}+u_{y y}^{i}+u_{z z}^{i}-A_{i}\right)\left(S_{n_{i}}^{+} S_{n_{i}}^{-}+S_{n_{i}}^{-} S_{n_{i}}^{+}\right)+\left(C_{1}^{i}+C_{2}^{i}\right)\left(S_{n_{i}}^{+} S_{n_{i}}^{\xi}+S_{n_{i}}^{\xi} S_{n_{i}}^{+}\right)+\text {e.g. }\right\}
\end{gathered}
$$

Here we denote

$$
\begin{array}{cc}
S_{n_{i}}^{ \pm}=S_{n_{i}}^{\eta} \pm i S_{n_{i}}^{\xi}, & \left\langle S_{n_{1}}^{\zeta}\right\rangle=\left\langle S_{n_{2}}^{\zeta}\right\rangle=\left\langle S^{\zeta}\right\rangle, \\
H_{i}=H \cos \theta_{i}-\left\langle S^{\zeta}\right\rangle\left[J_{0} \cos 2 \psi+D_{0} \sin 2 \psi\right], & \bar{H}_{i}=\frac{1}{2}\left\{H \sin \theta_{i}-\left\langle S^{\zeta}\right\rangle\left[J_{0} \sin 2 \psi-D_{0} \cos 2 \psi\right]\right\}, \\
A_{i}=u_{x x}^{i} \cos ^{2} \theta_{i}+u_{y y}^{i} \sin ^{2} \theta_{i}+(-1)^{i+1} u_{x y}^{i} \sin 2 \theta_{i}, & B_{i}=-2 i\left\{u_{y z}^{i} \cos \theta_{i}+(-1)^{i+1} u_{x z}^{i} \sin \theta_{i}\right\}, \\
C_{1}^{i}=(-1)^{i}\left(u_{x x}^{i}-u_{y y}^{i}\right) \sin 2 \theta_{i}+2 u_{x y}^{i} \cos 2 \theta_{i}, & C_{2}^{i}=-2 i\left\{u_{x z}^{i} \cos \theta_{i}+(-1)^{i+1} u_{y z} \sin \theta_{i}\right\},
\end{array}
$$

where $J_{0}$ is the zero Fourier component of the exchange integral, and $D_{0}$ is the zero Fourier component of the Dzyaloshinskii exchange. Here

$$
J_{0}=\sum_{n} J(n)
$$

Solving the one-ion problem with the Hamiltonian (3)

$$
\mathcal{H}_{0}\left(n_{i}\right) \psi_{n_{i}}(M)=E_{M}^{i} \psi_{n_{i}}(M)
$$


we determine the eigenvalues and eigenfunctions of $\mathcal{H}_{0}$ ( $M$ is the magnetic quantum number. For $S=1$ $M=-1,0,1)$ :

$$
\begin{aligned}
E_{1,-1}^{i}= & \frac{\beta}{4}+\frac{v / 2}{H_{i}^{2}+4 \bar{H}_{i}^{2}}\left\{\left(u_{x x}^{i}+u_{y y}^{i}\right)\left(H_{i}^{2}+8 H_{i}^{2}\right)+u_{z z}^{i}\left(H_{i}^{2}+4 \bar{H}_{i}^{2}\right)+A_{i}\left(H_{i}^{2}-4 \bar{H}_{i}^{2}\right)+(-1)^{i+1} 2 C_{1}^{i} H_{i} \bar{H}_{i}\right\} \overline{+} \\
\mp & \left\{\frac{\beta^{2}}{16}+H^{2}+4 \bar{H}_{i}^{2}-\frac{v \beta / 4}{H_{i}^{2}+4 \bar{H}_{i}^{2}}\left(u_{x x}^{i}+u_{y y}^{i}\right) H_{i}^{2}-u_{z z}^{i}\left(H_{i}^{2}+4 \bar{H}_{i}^{2}\right)-A_{i}\left(H_{i}^{2}-4 \bar{H}_{i}^{2}\right)+(-1)^{i+1} 2 C_{1}^{i} H_{i} \bar{H}_{i}\right]+ \\
& \left.+\left(\frac{v / 2}{H_{i}^{2}+4 \bar{H}_{i}^{2}}\right)\left[\left(u_{x x}^{i}+u_{y y}^{i}\right) H_{i}^{2}-u_{z z}^{i}\left(H_{i}^{2}+4 \bar{H}_{i}^{2}\right)-A_{i}\left(H_{i}^{2}-4 \bar{H}_{i}^{2}\right)+(-1)^{i+1} 2 C_{1}^{i} H_{i} \bar{H}_{i}\right]^{2}\right\} \\
& E_{0}^{i}=\frac{\beta}{2}+\frac{v}{H_{i}^{2}+4 \bar{H}_{i}^{2}}\left\{\left(u_{x x}^{i}+u_{y y}^{i}\right) H_{i}^{2}+u_{z z}^{i}\left(H_{i}^{2}+4 \bar{H}_{i}^{2}\right)-A_{i}\left(H_{i}^{2}-4 \bar{H}_{i}^{2}\right)+(-1)^{i+1} 2 C_{1}^{i} H_{i} \bar{H}_{i}\right\} .
\end{aligned}
$$

The Hubbard's operators are built on eigenfunctions by standard rules [9] $Y_{n}^{M^{\prime} M}=\left|\psi_{n}\left(M^{\prime}\right)\right\rangle \mid\left\langle\psi_{n}(M)\right|$ and describe the transition of a magnetic ion state $M$ to state $M^{\prime}$. These operators are ${ }^{i}$ related to spin operators through familiar relations [9].

From the condition of free energy density minimum

$$
\mathcal{F}=\mathcal{F}_{0}-T \ln Z,
$$

where $\mathcal{F}_{0}$ is the free energy of an elastics subsystem which is determined by the last term in (1)

$$
Z=\sum_{M} \exp \left(-E_{M} / T\right)
$$

and $Z$ is the partition sum. Determine the equilibrium (spontaneous) deformations $u_{j k}^{(0)}\left(n_{i}\right)$. They appear to be

$$
\begin{gathered}
u_{x x}^{(0) i}=\frac{b_{1}^{i}(\eta+2 \lambda)-\lambda\left(b_{2}^{i}+b_{3}^{i}\right)}{\eta(\eta+3 \lambda)}, u_{y y}^{(0) i}=\frac{b_{2}^{i}(\eta+2 \lambda)-\lambda\left(b_{1}^{i}+b_{3}^{i}\right)}{\eta(\eta+3 \lambda)}, u_{z z}^{(0) i}=\frac{b_{3}^{i}(\eta+2 \lambda)-\lambda\left(b_{1}^{i}+b_{2}^{i}\right)}{\eta(\eta+3 \lambda)}, \\
u_{x z}^{(0) i}=u_{y z}^{(0) i}=0, \quad u_{x y}^{(0) i}=\frac{(-1)^{i+1}}{2 \eta} \frac{v\left(H_{i}^{2}-4 \bar{H}_{i}^{2}\right) \sin ^{2}\left(\psi+(-1)^{i+1} \varphi\right)-4 H_{i} \bar{H}_{i} \cos \left(\psi+(-1)^{i+1} \varphi\right)}{H_{i}^{2}+4 \bar{H}_{i}^{2}} \times \\
\times\left\{\cosh \frac{\kappa_{i}^{0}}{T}+\frac{\beta}{\kappa_{i}^{0}} \sinh \frac{\kappa_{i}^{0}}{T}-\exp (-\beta / 4 T)\right\} / Z_{i}^{(0)},
\end{gathered}
$$

where

$$
\begin{aligned}
& b_{1}^{i}=-P-\frac{v}{Z_{i}^{(0)}}\left\{\left[1-\frac{\left(H_{i} \cos \left(\psi+(-1)^{i+1} \varphi\right)+2 \bar{H}_{i} \sin \left(\psi+(-1)^{i+1} \varphi\right)\right)^{2}}{H_{i}^{2}+4 \bar{H}_{i}^{2}}\right] \cosh \frac{\kappa_{i}^{0}}{T}-\right. \\
& \left.-\frac{\left[H_{i} \sin \left(\psi+(-1)^{i+1} \varphi\right)-2 \bar{H}_{i} \cos \left(\psi+(-1)^{i+1} \varphi\right)\right]^{2}}{H_{i}^{2}+4 \bar{H}_{i}^{2}}\left(\frac{\beta}{4 \kappa_{i}^{0}} \sinh \frac{\kappa_{i}^{0}}{T} \mathrm{e}^{-\beta / 4 T}\right)\right\}, \\
& b_{2}^{i}=-\frac{v}{Z_{i}^{0}}\left\{\left[1+\frac{\left(H_{i} \sin \left(\psi+(-1)^{i+1} \varphi\right)+2 \bar{H}_{i} \cos \left(\psi+(-1)^{i+1} \varphi\right)\right)^{2}}{H_{i}^{2}+4 \bar{H}_{i}{ }^{2}}\right] \cosh \frac{\kappa_{i}^{0}}{T}-\right. \\
& \left.-\frac{\left(H_{i} \cos \left(\psi+(-1)^{i+1} \varphi\right)+2 \bar{H}_{i} \sin \left(\psi+(-1)^{i+1} \varphi\right)\right)^{2}}{H_{i}^{2}+4 \bar{H}_{i}^{2}}\left(\frac{\beta}{4 \kappa_{i}^{0}} \cosh \frac{\kappa_{i}^{0}}{T}-\mathrm{e}^{-\beta / 4 T}\right)\right\}, \\
& b_{3}^{i}=-\frac{v}{Z_{i}^{(0)}}\left\{\cosh \frac{\kappa_{i}^{0}}{T}+\frac{\beta}{4 \kappa_{i}^{0}} \sinh \frac{\kappa_{i}^{0}}{T}+\mathrm{e}^{-\beta / 4 T}\right\}, \quad Z_{i}^{(0)}=2 \cosh \frac{\kappa_{i}^{0}}{T}+\mathrm{e}^{-\beta / 4 T}, \quad \kappa_{i}^{0}=\left\{H_{i}^{2}+4 \bar{H}_{i}^{2}+\frac{\beta^{2}}{16}\right\}^{1 / 2} .
\end{aligned}
$$


As is evident from (4) and (6), the energy level corresponding to the ground state is $E$, whose analytical expression in our approximation is

$$
\begin{gathered}
E_{1}^{i}=-\frac{H_{i}^{2}+4 \bar{H}_{i}^{2}}{\beta}-\frac{v P}{\eta}+ \\
+\frac{2 v P}{\eta} \frac{H_{i} \bar{H}_{i}}{H_{i}^{2}+4 \bar{H}_{i}^{2}} \sin 2\left(\psi+(-1)^{i+1} \varphi\right) .
\end{gathered}
$$

In (8) the terms proportional to $v^{2} / \eta$ are rather cumbersome and we will omit them here. Near the phase-transition lines these expressions are important and we take them explicitly into account.

In this case the free energy density can be represented in the form

$$
\begin{gathered}
\mathcal{F}^{i}=\mathcal{F}_{0}^{i}+E_{1}^{i} \\
\mathcal{F}_{0}^{i}=\frac{\lambda+\eta}{2}\left(H_{i}^{2}+u_{y y}^{2}+u_{z z}^{2}\right)+\eta\left(u_{x y}^{2}+u_{x z}^{2}+u_{y z}^{2}\right)+ \\
+\lambda\left(u_{x x} u_{y y}+u_{x x} u_{z z}+u_{y y} u_{z z}\right)+P u_{x x}
\end{gathered}
$$

Using (9), we determine the phases in which the system under study may exist. Suppose that the system has such values of field $H$ and pressure $P$ that the mean magnetization at the sites is oriented as shown in Fig. 1. With an increase in the field to a certain value $H_{c 2}$ (at constant pressure), the vector of mean magnetization is oriented along the direction of the magnetic field $H$. In this case the angle $\varphi=0$ and $\psi$ is determined from the condition of free energy density minimum (9). This condition leads to that in a phase with $\psi=0, \bar{H}_{i}=0$ from which it is easy to obtain

$$
\cos \psi=\frac{H+\left\langle S^{\xi}\right\rangle\left|D_{0}\right|}{2 J_{0}\left\langle S^{\xi}\right\rangle} .
$$

One can demonstrate that in the $H_{c 2}$ vicinity the mean sublattice magnetization approximately equals unity, $\left\langle S^{\xi}\right\rangle=1$. The phase which is realized at $H \geq H_{c 2}$ is called the FM1 phase. We determine the value of $H_{c 2}$ from the ME wave spectra.

A further increase of the field leads to the fact that the vector $\mathbf{M}=\mathbf{M}_{1}+\mathbf{M}_{2}$ orients along the direction of the field (i.e., $\varphi=\psi=0$ ) as well as vectors of sublattice magnetization. Such configuration occurs at $H \geq H_{c 3}$, where $H_{c 3}=2 J_{0}-\left|D_{0}\right|$.

The phase realized at $H \geq H_{c 3}$ is called the FM2 phase. Of most interest is the case of small fields in which the so-called quadruple (QU) phase is realized. In this phase the magnetic vector order parameter equals zero [7].

For our system it means that the mean sublattice magnetization and the mean magnetization at the site equal zero. It is well known [7] that for easy-plane ferromagnets in a transverse magnetic field the realization of a $\mathrm{QU}$ phase is accompanied by the inversion of energy levels; i.e., the $E_{0}$ level becomes the lowest level. In the system under investigation there is no such inversion and the QU phase is realized due to certain purely quantum effects.

Let us discuss this question in detail. Let us assume that in a certain field the system indergoes a transition to the $\mathrm{QU}$ phase. The magnetic phase realized in fields $H_{c 2} \leq H \leq H_{c 1}$ is called the QFM phase (quadruple ferromagnet). In our geometry the pressure plays the role of an effective anisotropy with the easy magnetization axis (EMA) parallel to the OY axis [6]. At fields close to the field of the QFM-QU phase transition, as follows from the analysis of the free energy density [9] its minimum corresponds to values of the angles and is close to the following: $\varphi=\pi / 2, \psi=0$; i.e., the vector of mean magnetization at the site the vectors of the sublattice mean magnetization tend to «turn» along the «effective» EMA. For mean magnetization of sublattices we obtain

$$
\left\langle S_{n_{i}}^{\xi}\right\rangle=4 \frac{H}{\beta} \cos \left(\psi+(-1)^{2(i+1)} \varphi\right) .
$$

At $P=0$ and $H=0$ the system is in the $\mathrm{QU}$ phase since this result may be interpreted in the following way: there is no distinct axis in the system and all the directions in the basic plane $(X Y)$ are equal. Therefore, magnetizations at different sites may be directed arbitrarily and their average equals zero. This situation is similar to the one in which the QU phase is realized in strongly anisotropic easy-plane ferromagnets [7].

As is evident, the ground state of an AFM at $P=0$ and $H=0$ corresponds to the eigenvector $|1\rangle$ of the $S^{\xi}$ operator:

$$
\psi_{n_{i}}(1)=|1\rangle .
$$

With increasing pressure the mean magnetization should be turned along the axis of the «effective» anisotropy (OY). The ground state of an AFM is a superposition of the eigenvectors $|1\rangle$ and $|-1\rangle$ of the $S^{\zeta}$ operator: $\psi_{n}(1)=\cos \delta_{i}|1\rangle+\sin \delta_{i}|-1\rangle$. Such superposition of the vectors $|1\rangle$ and $|-1\rangle$ leads to the quantum redaction of the spin. This effect is characteristic of easy-axis magnets in a magnetic field 


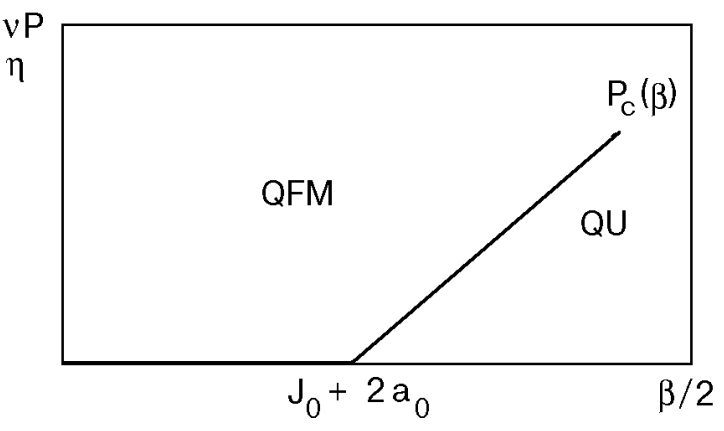

Fig. 2. Phase diagram of an easy-plane AFM in the plane $(P, \beta)$.

perpendicular to an easy axis. In our case this effect is caused by the presence of an external pressure, which plays the role of uniaxial anisotropy.

In the AFM under study the two described quantum effects account for the existence of the QU phase. It turns out that the QU phase exists up to pressures determined by the formula

$$
\frac{\nu P_{c}}{\eta}=\frac{\beta}{2}-J_{0}-2 a_{0},
$$

where

$$
a_{0}=v^{2} / 2 \eta
$$

This expression can be obtained by investigating the existence domain of a phase with nonzero mean magnetization. In Fig. 2 we show the phase diagram at $H=0$ in the plane $(P, \beta)$. The field of QFM-QU phase transition $\left(H_{c 1}\right)$ can be determined from the ME wave spectra. The behavior of angles and sublattice magnetization as a function of external magnetic field is shown in Figs. 3 and 4, where we denote

$$
\psi_{0}=\frac{D_{0}}{4 J_{0}}+\left(\frac{D_{0}^{2}}{16 J_{0}^{2}}+\frac{d_{0}}{2 J_{0}}\right)^{1 / 2}, d_{0}=P v / \eta
$$

\section{Spectra of bound ME waves}

The dynamic properties of magnets have several special features in the vicinity of orientational phase transitions. It is well known that allowance for the $\mathrm{ME}$ interactions leads to hybridization of elementary excitation [3,6]. Although the ME exchange is weak in the vicinity of OPT, precisely this parameter plays the decisive role in the dynamics of a system $[3,6]$. To investigate this question we represent the deformation tensor as a sum of two terms: spontaneous deformations $u_{j k}^{(0)}\left(n_{i}\right)$, which are determined by (6), and the dynamic term $u_{j k}^{(1)}\left(n_{i}\right)$, which corresponds which to the lattice oscillations.

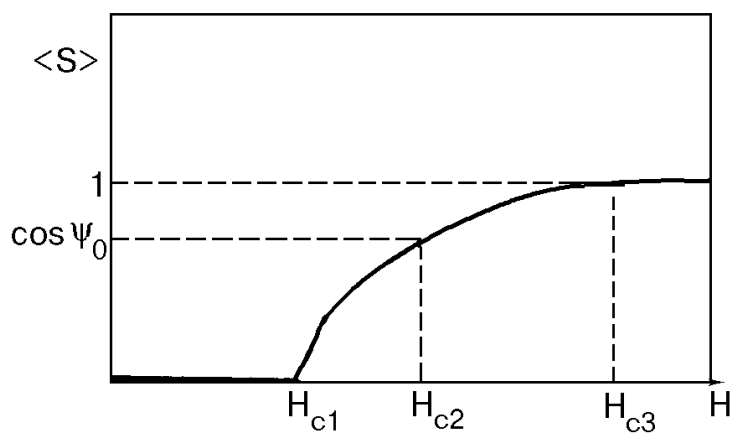

Fig. 3. Dependence of the sublattice magnetization on the external magnetic field.

The latter may be written in terms of phonon operators as follows [8]:

$$
u_{j k}^{(1) i}=\frac{i}{2} \times
$$

$\times \sum_{q, \lambda} \frac{\exp \left(i q n_{i}\right)}{\left(2 m N \omega_{\lambda}(q)\right)^{1 / 2}}\left(b_{q, \lambda}+b_{-q, \lambda}^{+}\right)\left(e_{\lambda}^{j}(q) q_{k}+e_{\lambda}^{k}(q) q_{j}\right)$

where $e_{\lambda}(q)$ is the unit vector of a phonon polarization, $\lambda=l, t, \tau ; m$ is the mass of a magnetic ion, $N$ is the number of sites in a lattice, $\omega_{\lambda}(q)=c_{\lambda} q$ is
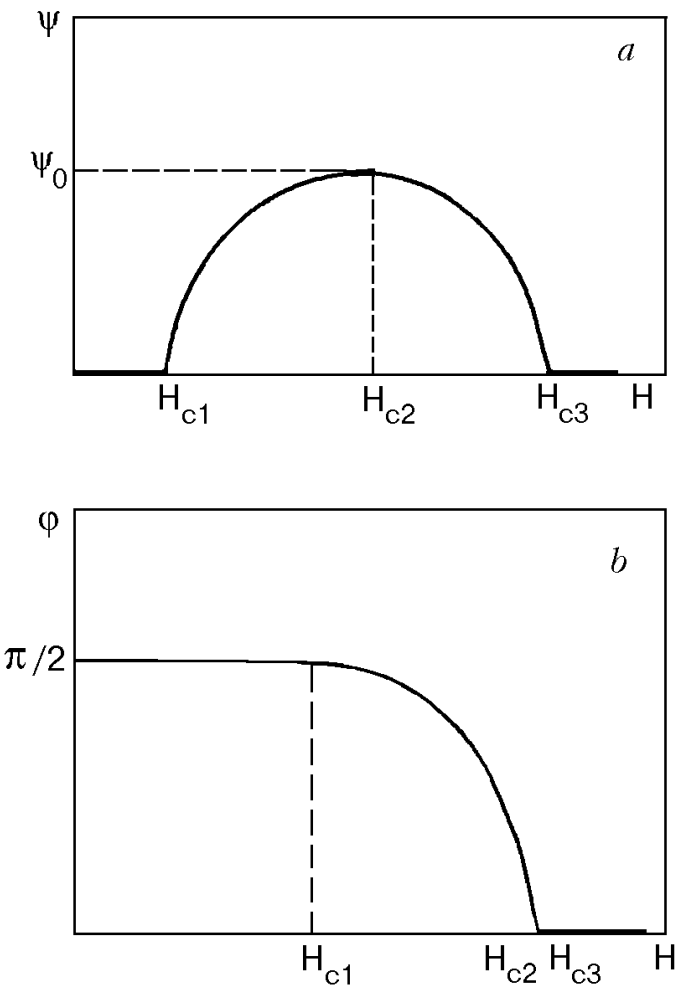

Fig. 4. Dependence of the angles of sublattice orientation on the external magnetic field. 
the dispersion of a free phonon, and $c_{\lambda}$ is the velocity of polarized sound.

After separation in the one-site Hamiltonian (3) of a part proportional to $u_{j k}^{(1)}\left(n_{i}\right)$ and its quantization in accordance with the given formula it is possible to write the Hamiltonian describing the processes of magnon-phonon transformations:

$$
\mathcal{H}_{t r}=\sum_{n_{i}}\left\{\sum_{M} \mathcal{P}_{M} J_{n_{i}}^{M}+\sum_{\alpha} \mathcal{P}_{\alpha}^{i} Y_{n_{i}}^{\alpha}\right\}
$$

where

$$
\mathcal{P}_{m(\alpha)}^{i}=\frac{1}{\sqrt{N}} \sum_{q, \lambda}\left(b_{q, \lambda}+b_{q, \lambda}^{+}\right) T_{n_{i}}^{M(\alpha)}(q, \lambda),
$$

$\alpha$ are the root vectors [6,9], and $T_{n_{i}}^{M(\alpha)}(q, \lambda)$ are the amplitudes of transformations

Further, we consider the simplest and the most interesting case in which the wave vector is parallel to an external field $H$. In such geometry the only nonzero components of the unit vector of phonon polarization are $e_{l}^{x}(q), e_{t}^{y}(q)$, and $e_{\tau}^{z}(q)$ and the corresponding amplitudes of transformation have the form

$$
\begin{gathered}
T_{n_{i}}^{1-1}(q, l)=T_{n_{j}}^{-11}(q, l)=\frac{i v}{2} T_{n_{j}}^{0}(q, l) q e_{l}^{x}\left(\frac{H_{j}}{\kappa_{j}^{0}} \cos 2 \delta_{i}-\frac{\beta}{4 \kappa_{i}^{0}} \sin 2 \delta_{i}\right) \sin ^{2} \psi \\
T_{n_{j}}^{10}(q, l)=T_{n_{j}}^{01}(q, l)=(-1)^{j+1} \frac{i v}{4} T_{n_{j}}^{0}(q, l) q e_{l}^{x}\left(\gamma_{\perp}^{j}\left(\alpha_{1}\right)-\gamma_{\perp}^{j}\left(\alpha_{2}\right)\right) \sin 2 \psi \\
T_{n_{j}}^{-10}(q, l)=T_{n_{j}}^{0-1}(q, l)=(-1)^{j+1} \frac{i v}{4} T_{n_{j}}^{0}(q, l) q e_{l}^{x}\left(\gamma_{\perp}^{j}\left(\alpha_{5}\right)-\gamma_{\perp}^{j}\left(\alpha_{6}\right)\right) \sin 2 \psi, \\
T_{n_{j}}^{1-1}(q, t)=T_{n_{j}}^{-11}(q, t)=-\frac{i v}{4} T_{n_{j}}^{0}(q, t) q e_{t}^{y}\left(\frac{H_{i}}{\kappa_{j}^{0}} \cos 2 \delta_{j}-\frac{\beta}{4 \kappa_{j}^{0}} \sin 2 \delta_{j}\right) \sin 2 \psi, \\
T_{n_{j}}^{10}(q, t)=T_{n_{j}}^{01}(q, t)=(-1)^{j+1} \frac{i v}{4} T_{n_{j}}^{0}(q, t) q e_{t}^{y}\left(\gamma_{\perp}^{j}\left(\alpha_{1}\right)-\gamma_{\perp}^{j}\left(\alpha_{2}\right)\right) \cos 2 \psi \\
T_{n_{j}}^{-10}(q, t)=T_{n_{j}}^{0-1}(q, t)=(-1)^{j+1} \frac{i v}{4} T_{n_{j}}^{0}(q, t) q e_{t}^{y}\left(\gamma_{\perp}^{j}\left(\alpha_{5}\right)-\gamma_{\perp}^{j}\left(\alpha_{6}\right)\right) \cos 2 \psi \\
T_{n_{j}}^{1-1}(q, \tau)=-T_{n_{j}}^{1-1}(q, \tau)=(-1)^{j} \frac{v}{2} T_{n_{j}}^{0}(q, \tau) q e_{\tau}^{z} \sin \psi \\
T_{n_{j}}^{10}(q, \tau)=-T_{n_{j}}^{01}(q, \tau)=\frac{v}{4} T_{n_{j}}^{0}(q, \tau) q e_{\tau}^{z}\left(\gamma_{\perp}^{j}\left(\alpha_{1}\right)+\gamma_{\perp}^{j}\left(\alpha_{2}\right)\right) \cos \psi \\
T_{n_{j}}^{-10}(q, \tau)=-T_{n_{j}}^{0-1}(q, \tau)=\frac{v}{4} T_{n_{j}}^{0}(q, \tau) q e_{\tau}^{z}\left(\gamma_{\perp}^{j}\left(\alpha_{5}\right)+\gamma_{\perp}^{j}\left(\alpha_{6}\right)\right) \cos \psi \\
T_{n_{j}}^{0}(q, \lambda)=\exp \left(i q n_{j}\right) / \sqrt{2 m \omega_{\lambda}(q)} .
\end{gathered}
$$

The functions $\gamma_{\perp}^{j}(\alpha)$ in (12) are determined from the relation between the spin operators and the Hubbard operators [6] and in the given geometry $\gamma_{j}^{j}(\alpha) \gamma_{\|}^{j}(\alpha)=0$ for all $\alpha, n$ is the number of the site, and $j$ is the number of the sublattice.

The spectra of ME waves are determined by the poles of a full Green's function

$$
G^{\alpha \alpha^{\prime}}\left(n, \tau ; n^{\prime}, \tau^{\prime}\right)=-\left\langle\hat{T} \tilde{Y}_{n}^{\alpha}(\tau) \tilde{Y}_{n^{\prime}}^{-\alpha}\left(\tau^{\prime}\right)\right\rangle,
$$

where $\hat{T}$ is the time-ordering operator, and $Y_{n}^{\alpha}(\tau)$ are the Hubbard operators in the Heisenberg representation.

The Fourier transform of the Green's function to be found is related to the irreducible transform by the Larkin part $\Sigma_{j j}^{\alpha \alpha^{\prime}}\left(k, \omega_{n}\right)$ and the amplitudes of transformation $T_{j}^{\alpha}(k, \lambda)$ are related by the Larkintype equation

$G_{j j^{\prime}}^{\alpha \alpha^{\prime}}\left(k, \omega_{n}\right)=\Sigma_{j j^{\prime}}^{\alpha \alpha^{\prime}}\left(k, \omega_{n}\right)+\left(\Sigma_{j}^{\alpha}\left(k, \omega_{n}\right) v(k) \mathbf{P}_{j^{\prime}}^{\alpha}\left(k, \omega_{n}\right)+\right.$

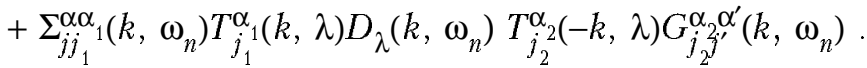

In (13) $D_{\lambda}\left(k, \omega_{n}\right)=2 \omega_{\lambda}(k) /\left(\omega_{n}^{2}-\omega_{\lambda}^{2}(k)\right)$ is the Green's function of a $\lambda$-polarized free phonon, and $\Sigma_{j}^{\alpha}\left(k, \omega_{n}\right)$ is the six-dimensional vector, which in the block notation has the form

$$
\Sigma_{j}^{\alpha}\left(k, \omega_{n}\right)=\left(\Sigma_{j 1}^{\alpha}\left(k, \omega_{n}\right), \Sigma_{2 j}^{\alpha}\left(k, \omega_{n}\right)\right),
$$


where the three-dimensional vectors $\Sigma_{j j^{\prime}}^{\alpha}\left(k, \omega_{n}\right)$ have the form

$$
\begin{gathered}
\sum_{j j^{\prime}}^{\alpha}\left(k, \omega_{n}\right)=\Sigma_{\alpha_{1}} \mathbf{c}_{j^{\prime}}\left(-\alpha_{1}\right) \sum_{j j^{\prime}}^{\alpha \alpha}\left(k, \omega_{n}\right), \\
\mathbf{c}_{j}(\alpha)=\left(\gamma_{\|}^{j}(\alpha), \gamma_{\perp}^{j}(\alpha), \gamma_{\perp}^{* j}(-\alpha)\right) .
\end{gathered}
$$

The six-dimensional matrix is $\hat{V}(k)=\sigma^{1} \otimes V$, and $\sigma^{1}$ is the Pauli matrix

$$
\begin{gathered}
V=\left(\begin{array}{ccc}
V^{\zeta \xi} & -V^{+\xi} & -V^{+\xi} \\
V^{+\xi} & V^{++} & V^{+-} \\
V^{+\xi} & V^{+-} & V^{++}
\end{array}\right) \\
V^{\zeta \xi}\left(n-n^{\prime}\right)=J\left(n-n^{\prime}\right) \cos 2 \psi+D\left(n-n^{\prime}\right) \sin 2 \psi, \\
V^{++}\left(n-n^{\prime}\right)=\frac{1}{4}\left\{V^{\xi \xi}\left(n-n^{\prime}\right)-J\left(n-n^{\prime}\right)\right\}, \\
V^{+-}\left(n-n^{\prime}\right)=\frac{1}{4}\left\{V^{\xi \xi}\left(n-n^{\prime}\right)+J\left(n-n^{\prime}\right)\right\},
\end{gathered}
$$

$V^{+\xi}\left(n-n^{\prime}\right)=\frac{1}{2}\left\{J\left(n-n^{\prime}\right) \sin 2 \psi-D\left(n-n^{\prime}\right) \cos 2 \psi\right\}$.

The six-dimensional vectors $\mathbf{P}_{j^{\prime}}^{\alpha}\left(k, \omega_{n}\right)$ can also be represented in a block notation

$$
\mathbf{P}_{j^{\prime}}^{\alpha}\left(k, \omega_{n}\right)=\left(P_{j 1}^{\alpha}\left(k, \omega_{n}\right), P_{2 j}^{\alpha}\left(k, \omega_{n}\right)\right),
$$

while the three-dimensional vectors $\mathbf{P}_{j j^{\prime}}^{\alpha}\left(k, \omega_{n}\right)$ are determined by the relation

$$
\mathbf{P}_{j j^{\prime}}^{\alpha}\left(k, \omega_{n}\right)=\sum_{\alpha_{1}} \mathbf{c}_{j}\left(\alpha_{1}\right) G_{j j^{\prime}}^{\alpha \alpha_{1}\left(k, \omega_{n}\right)}
$$

Equation (13) can be solved due to the «split» dependence on index $\alpha$. Finally, the dispersion relation in the mean-field approximation for the ME waves has the form

$$
\left\{1-\left(y_{2}-y_{3}\right)^{2}\right\}\left\{1-\left(y_{2}+y_{3}\right)^{2}\right\}=0
$$

where

$$
\begin{gathered}
y_{2}\left(k, \omega_{n}\right)=-V^{++}(k)\left\{b(\alpha) G_{0}^{\alpha}\left(\omega_{n}\right)\left|\gamma_{\perp}(\alpha)\right|^{2}+\frac{D_{\lambda}\left(k, \omega_{n}\right)}{1-Q_{\lambda \lambda^{\prime}} D_{\lambda}\left(k, \omega_{n}\right)} b(\alpha) b(\beta) G_{0}^{\alpha}\left(\omega_{n}\right) \gamma_{\perp}(\alpha) \gamma_{\perp}^{*}(\beta) T^{\alpha}(k, \lambda) T^{\beta}\left(-k, \lambda^{\prime}\right)\right\}, \\
y_{3}\left(k, \omega_{n}\right)= \\
=V^{+-}(k)\left\{b(\alpha) G_{0}^{\alpha}\left(\omega_{n}\right) \gamma_{\perp}(\alpha) \gamma_{\perp}(-\alpha)+\frac{D_{\lambda}\left(k, \omega_{n}\right)}{1-Q_{\lambda \lambda^{\prime}} D_{\lambda}\left(k, \omega_{n}\right)} b(\alpha) b(\beta) G_{0}^{\alpha}\left(\omega_{n}\right) G_{0}^{\beta}\left(\omega_{n}\right) \gamma_{\perp}(\alpha) \gamma_{\perp}(-\beta) T^{-\alpha}(k, \lambda) T^{-\beta}\left(-k, \lambda^{\prime}\right)\right\}, \\
Q_{\lambda \lambda^{\prime}}=T_{j}^{\alpha}\left(-k, \lambda^{\prime}\right) \Sigma_{j j^{\prime}}^{\alpha \alpha^{\prime}}\left(k, \omega_{n}\right) T_{j^{\prime}}^{-\alpha^{\prime}}(k, \lambda)
\end{gathered}
$$

Here we sum over $\alpha, \beta, \lambda$, and $\lambda^{\prime}$.

As is evident from the analysis of (14) in the QFM phase, the transverse polarized ME waves ( $\tau$ and $t$ ) «become entangle», while the equation for the longitudinally polarized wave splits out (1) and its spectra has the form

$$
\omega_{1}^{2}(k)=\omega_{l}^{2}(k)\left(1-a_{0} / J_{0}\right) .
$$

However, in the vicinity of the OPT QFM-FM phase and QFM-QU phase it is possible to uncouple Eq. (14) with respect to polarizations. Let us consider the dynamics of a system in the vicinity of the QFM-FM1 phase transition. In this case (see above) $\varphi=0, \cos 2 \psi \approx-1$, and $\cos \psi \approx 0$, while the mean sublattice magnetization is $\left\langle S_{i}^{\xi}\right\rangle \approx 1$. It follows from (12) that the only nonzero amplitudes of transformations are $T^{10}(k, t)$ and $T^{01}(k, t)$ and only $t$-polarized phonons interact with the magnetic subsystem. The equation $1-\left(y_{2}+y_{3}\right)^{2}=0$ decomposes in the vicinity of the QFM-FM1 transition into three equations, each describing quasiphonon oscillations of certain polarization. The solutions of these equations are

$$
\begin{gathered}
\omega_{\mathrm{I}}^{2}(k)=\omega_{l}^{2}(k)\left(1-a_{0} / J_{0}\right), \\
\omega_{\mathrm{II}}^{2}(k)=\omega_{\tau}^{2}(k)\left(1-a_{0} / 4 J_{0}\right), \\
\omega_{\mathrm{III}}^{2}(k)=\omega_{t}^{2}(k) \frac{\alpha k^{2}+\left[\left(H^{2}+H\left|D_{0}\right|\right) / 2 J_{0}\right]-d_{0}}{\alpha k^{2}+a_{0}+\left[\left(H^{2}+H\left|D_{0}\right|\right) / 2 J_{0}\right]-d_{0}} .
\end{gathered}
$$

From the condition $\left[\left(H^{2}+H\left|D_{0}\right|\right) / 2 J_{0}\right]-d_{0}=0$ we determine the field of a QFM-FM1 phase transition

$$
H_{c 2}=-\frac{\left|D_{0}\right|}{2}+\left(\frac{D_{0}^{2}}{4}-2 d_{0} J_{0}\right)^{1 / 2}
$$


It follows from (16) that at the point of OPT in a long-wave limit $\left(\alpha k^{2}<<a_{0}\right)$ the spectrum of $t$-polarized quasiphonons softens:

$$
\begin{gathered}
\omega_{\mathrm{I}}(k) \approx \omega_{l}(k), \\
\omega_{\mathrm{II}}(k) \approx \omega_{\tau}(k), \\
\omega_{\mathrm{III}}^{2}(k)=\omega_{t}^{2}(k) \frac{\alpha k^{2}}{a_{0}},
\end{gathered}
$$

while the spectrum of quasimagnons is determined by the expression

$$
\mathcal{E}^{2}(k)=2 J_{0}\left(\alpha k^{2}+a_{0}\right) .
$$

Thus $t$-polarized phonons strongly interact with magnons in the vicinity of the OPT (QFM-FM1). Their spectrum softens while in quasimagnon spectrum the ME gap appears.

The value of the gap, as follows from (19), is

$$
\omega_{m e}=\sqrt{2 J_{0} a_{0}} .
$$

The results (18)-(20) obtained by us precisely coincide with the results for slightly anisotropic AFM [6].

Of special interest is the dynamics of a system in the vicinity of the OPT QFM-QU phase since this case has not been investigated previously.

As we have noticed above, near the point under study $\varphi=\pi / 2$ and $\psi=0$, while the mean sublattice magnetization equals zero: $\left\langle S^{\zeta}\right\rangle \approx 0$. Hence $V^{++}=0$ and therefore $y_{2}$ equals zero (15). The dispersion relation (14) has a more simple form:

$$
1-y_{3}^{2}=0 \text {. }
$$

It should be noted that in this situation $T^{10}(k, \tau)$ and $T^{01}(k, \tau)$ amplitudes of transformation differ from zero $T^{10}(k, t) \approx T^{01}(k) \approx 0$ by virtue of infinitesimally small $H / \beta$ ratio. Equation (21) accordingly splits into equations, each of which describes quasiphonon oscillations of certain polarization. These equations have the solutions

$$
\begin{gathered}
\omega_{\mathrm{I}}^{2}(k)=\omega_{l}^{2}(k), \\
\omega_{\mathrm{II}}^{2}(k)=\omega_{\tau}^{2}(k) \frac{\alpha k^{2}+H_{c 1}^{2}-H^{2}}{\alpha k^{2}+H_{c 1}^{2}-H^{2}+2 a_{0}}, \\
\omega_{\mathrm{III}}^{2}(k) \approx \omega_{t}^{2}(k),
\end{gathered}
$$

where

$$
H_{c 1}=\sqrt{\beta / 2\left(\beta / 2-d_{0}-2 a_{0}-J_{0}\right)} .
$$

Here $H_{c 1}$ is the field of a OPT QFM-QU. At the point of a transition in a long-wave limit $\left(\alpha k^{2}<<a_{0}\right)$ the spectrum of $\tau$-polarized quasiphonons softens:

$$
\omega_{\mathrm{II}}^{2}(k)=\omega_{\tau}^{2}(k) \alpha k^{2} / 2 a_{0},
$$

while in the spectrum of quasimagnons

$$
\mathcal{E}^{2}(k)=\left(\alpha k^{2}+2 a_{0}\right)\left(2 J_{0}+2 a_{0}\right),
$$

the ME gap appears

$$
\omega_{m e}=2 \sqrt{J_{0} a_{0}} .
$$

As is evident from the relations (18) and (23), in the vicinity of the point of OPT QFM-QU phase the soft mode is a $\tau$-polarized quasiphonon mode, while at the point of OPT QFM-QU phase a $t$-polarized quasiphonon mode is a soft mode. Therefore, in the QFM phase (at $H_{c 2}<H<H_{c 1}$ ) two transversely polarized modes interact with the magnon branch.

Note that the value of ME gap in the spectrum of quasimagnons in the QU-phase is $\sqrt{2}$ times larger than the gap in the vicinity of a transition to the FM1 phase. Besides, as follows from (20) and (25), the ME gap suffers the so-called exchange amplification characteristic of antiferromagnets.

From the condition $H_{c 1}<H_{c 2}$ we obtain the value of a one-ion anisotropy constant at which the above-described effects are realized

$$
\begin{aligned}
& \beta>J_{0}+d_{0}+2 a_{0}+\left\{\left(J_{0}+d_{0}+2 a_{0}\right)^{2}+\right. \\
& \left.+D_{0}^{2}+4 d_{0}-4\left|D_{0}\right| \sqrt{D_{0}^{2} / 4+J_{0} d_{0}}\right\}^{1 / 2} .
\end{aligned}
$$

\section{Conclusions}

As we have noted recently, anisotropic magnets have attracted considerable attention. Peculiarities of their behavior were studied in many papers [1-3]. However, the influence of mechanical boundary conditions on the properties of such systems was virtually neglected. In the present paper it is demonstrated that the presence of mechanical boundary conditions (external pressure $P$ ) leads to a series of specific features in the behavior of easy-plane AFM.

In particular, these peculiarities exhibit themselves in the QU phase. We show that this phase is formed due to the presence of two quantum effects, one of which is characteristic only of the easy-plane magnets and the other is characteristic of the easy-axis magnets. 
Quite interesting is the effect involving the change of polarization of the soft mode, which softens in the transition from $H_{c 1}$ to $H_{c 2}$.

1. V. G. Borisenko and Yu. V. Pereverzev, Fiz. Nizk. Temp 11, 730 (1985).

2. W. G. Bos, T. O. Khassen, N. J. Poulis, and R. L. Carlin, Magn. Magn. Mater. 15-18, 464 (1980).

3. Yu. N. Mitsay and Yu. A. Fridman, Ukr. Fiz. Zh. 35, 459 (1990).

4. F. J. Varret, Phys. Chem. Solids 37, 257 (1976).
5. E. A. Zavadskii, Thesis of a report, Makhachkala (1984), p.131.

6. Yu. N. Mitsay and Yu. A. Fridman, Ukr. Fiz. Zh. 35, 5 (1990).

7. F. N. Onufriyeva, Fiz. Tverd. Tela 26, 145 (1984).

8. L. D. Landau and E. M. Lifshitz, Statistical Physics, Nauka, Moskow (1976).

9. R. O. Zaitsev, Zh. Eksp. Teor. Fiz. 63, 207 (1975). 